\title{
Treatment with Mesenchymal Stem Cells Improves Renovascular Hypertension and Preserves the Ability of the Contralateral Kidney to Excrete Sodium
}

\author{
Vanessa Araujo Varela ${ }^{a} \quad$ Elizabeth B. Oliveira-Sales ${ }^{a} \quad$ Edgar Maquigussa ${ }^{a}$ \\ Fernanda T. Borges ${ }^{a}$ Pedro P. Gattai ${ }^{a}$ Antonio da S. Novaes ${ }^{a}$ \\ Caroline G. Shimoura ${ }^{b}$ Ruy R. Campos ${ }^{b}$ Mirian A. Boim ${ }^{a}$ \\ ${ }^{a}$ Department of Medicine, Renal Division, Federal University of São Paulo, São Paulo, Brazil; \\ bDepartment of Physiology, Cardiovascular Division, Federal University of São Paulo, \\ São Paulo, Brazil
}

\section{Keywords}

Mesenchymal stem cells · Renovascular hypertension · Renal transporters · Cell therapy ·

Water channels · Sodium excretion

\begin{abstract}
Background: Mesenchymal stem cells (MSC) improve renal function and renovascular hypertension in the 2-kidney 1-clip model (2K-1C). While MSC play an immunomodulatory role, induce neoangiogenesis, and reduce fibrosis, they do not correct sodium loss by the contralateral kidney. Objectives: We investigated the tubular function of both stenotic and contralateral kidneys and the effect of MSC treatment by evaluating diuresis, natriuresis, and the expression of the main water and sodium transporters. Method: Adult Wistar rats were allocated into four groups: control (CT), CT+MSC, $2 \mathrm{~K}-1 \mathrm{C}$, and $2 \mathrm{~K}-1 \mathrm{C}+\mathrm{MSC}$. MSC $\left(2 \times 10^{5}\right)$ were infused through the tail vein 3 and 5 weeks after clipping. Systolic blood pressure (SBP) was monitored weekly by plethysmography. Six weeks after clipping, 24-hour urine and blood samples were collected for biochemical analysis. Gene expression of the $\mathrm{Na} / \mathrm{H}$ exchanger-3, epithelial sodium channel, $\mathrm{Na} / \mathrm{K}-\mathrm{ATPase}, \mathrm{Na} / \mathrm{K} / 2 \mathrm{Cl}$ cotransporter, and aquaporins 1 and 2 (AQP1 and AQP2) were analyzed by RT-PCR. Intrarenal distribution of AQP1 and AQP2 was analyzed by immunohistochemistry. Results: In hypertensive $2 \mathrm{~K}-1 \mathrm{C}$ animals, MSC prevented additional increases in BP. AQP1, but not AQP2, was suppressed in the contralateral kidney, resulting in significant increase in urinary flow rate and sodium excretion. Gene expressions of sodium transporters were similar in both kidneys, suggesting that the high perfusing pressure in the contralateral kidney was responsible for increased natriuresis. Contralateral hyper-
\end{abstract}




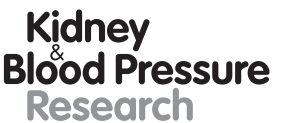

Research

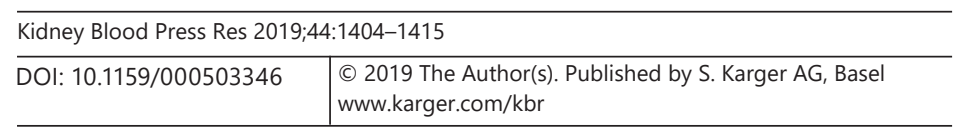

Varela et al.: Mesenchymal Stem Cells and Renovascular Hypertension

tensive kidney showed signs of renal deterioration with lower GFR in spite of normal RPF levels. Conclusions: MSC treatment improved renal function and enhanced the ability of the contralateral kidney to excrete sodium through a tubular independent mechanism contributing to reduce SBP.

(c) 2019 The Author(s)

Published by S. Karger AG, Basel

\section{Introduction}

Chronic renal artery stenosis is one of the most frequent causes of renovascular hypertension (RVH), which can lead to irreversible renal tissue injury and deterioration of renal function in consequence of chronic hypoxia. Moreover, the contralateral hypertensive kidney also presents signs of functional deterioration $[1,2]$.

The main mechanism involved in the genesis of RVH involves an initial activation of the renin-angiotensin system due to the decline in the renal perfusion pressure, followed by stimulation of the sympathetic nervous system contributing to maintain the RVH [3]. Renal artery stenosis results in variable degrees of renal hypoperfusion leading to chronic renal ischemia that may progress to irreversible kidney damage perpetuating RVH [4-7]. In contrast, the contralateral kidney is submitted to a high perfusion pressure that results in decreased renin secretion and it develops, over time, characteristics typical of a hypertensive kidney. In the early stages, contralateral kidney presents hyperfiltration, polyuria, and exaggerated natriuresis in response to the high perfusion pressure $[6,8,9]$. In addition, the suppression of renin-angiotensin system activity in the contralateral kidney can contribute to urinary sodium loss. Excessive natriuresis is currently believed to be one of the mechanisms involved in polyuria found in animals with RVH. On the other hand, less is known about the role of $\mathrm{Na}^{+}$ and water transporters in these events [8,10-12].

The recovery of renal function and structure after renal artery stenosis is still a challenge, since chronic renal ischemia produces structural and functional renal damage that may become irreversible and the revascularization may be insufficient to induce renal function recovery [13-15]. Thus, new therapies capable of repairing the post-stenotic kidney are required. In this context, stem cell therapy appears to be an attractive strategy. Oliveira-Sales and colleagues [1] showed that mesenchymal stem cells (MSC) blunted the progressive increase in blood pressure in rats with RVH induced by 2-kidney 1-clip model (2K-1C) with significant improvement of the renal architecture and of the microvascular rarefaction in the stenotic kidney with reduction in renal fibrosis in both kidneys [2]. In addition to these beneficial effects of MSC, we showed here that the treatment with MSC also improved the capacity of the contralateral kidney to excrete sodium.

Considering that the mechanisms that regulate the ability of the stenotic and contralateral kidneys to excrete sodium and water are not fully understood, the present work evaluated the expression of the major $\mathrm{Na}^{+}$and water transporters in animals with $\mathrm{RVH}$ in both the stenotic and contralateral kidneys and analyzed the effects of MSC on the expression of these transporters.

\section{Materials and Methods}

Young male Wistar rats (150-180 g) were supplied by the animal facility of the Federal University of São Paulo, São Paulo, Brazil. Rats were housed in collective cages, with free access to rat chow and tap water and maintained in a temperature-controlled environment $\left(23^{\circ} \mathrm{C}\right)$ on a 12 -hour light/dark cycle. 


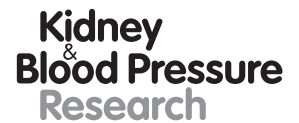

Kidney
Blood Pressure

Research

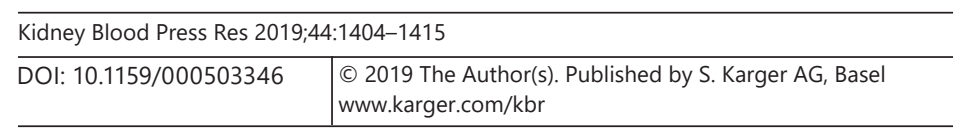

Varela et al.: Mesenchymal Stem Cells and Renovascular Hypertension

\section{Goldblatt Renovascular Hypertension Model (2K-1C)}

Animal model of RVH was induced by partial left renal artery obstruction as originally described by Goldblatt [16] (2-kidney 1-clip model). In brief, the animals were anesthetized with ketamine (Agener, União, Brazil - $40 \mathrm{mg} / \mathrm{kg}$ ) and xylazine (Sedafarm, Farmabase Saúde Animal Ldta, Brazil - $20 \mathrm{mg} / \mathrm{kg}$ ), and a 0.2-mm silver clip was implanted around the left renal artery in order to induce a $50 \%$ obstruction. Control animals (CT) were submitted to the same surgical procedure but with no renal artery occlusion. Animals were euthanized 6 weeks after clipping. The systolic blood pressure (SBP) was recorded by tail-cuff plethysmography every week.

\section{MSC Isolation, Expansion, and Characterization}

MSC were isolated from the tibias and femurs of male Wistar rats (150-180 g). Flushed material was subjected to a density gradient for isolation of mononuclear cells by Histopaque (Sigma-Aldrich, St. Louis, MO, USA). Cells were transferred to plastic dishes in low-glucose DMEM (Gibco, NY, USA) supplemented with 10\% FBS (Emcare, Campinas, Brazil). The culture flasks were kept in a $95 \%$ air and $5 \% \mathrm{CO}_{2}$ humidified environment at $37^{\circ} \mathrm{C}$ until the cells reached $70-80 \%$ confluence. Cells were then detached with trypsin and subsequently subcultured until the 6th passage. At the 2 nd passage, cells were subjected to osteogenic and adipocyte differentiation assays. For osteogenic differentiation, MSC were incubated in DMEM containing low glucose and 10\% FCS supplemented with dexamethasone $(0.1 \mu \mathrm{M})$, ascorbic acid $(0.2 \mathrm{mM})$, and beta glycerol phosphate $(10 \mathrm{mM})$ for 28 days and stained with alizarin. For adipocyte assay, cells were incubated with DMEM containing low glucose and 10\% FBS supplemented with dexamethasone $(1 \mu \mathrm{M})$, IBMX $(0.5 \mathrm{mM})$, insulin $(10 \mu \mathrm{g} / \mathrm{mL})$, and indomethacin $(100 \mu \mathrm{M})$ for 3 days, and then culture medium was changed to DMEM containing only insulin $(20 \mu \mathrm{g} / \mathrm{mL})$ for 21 days. Then, the differentiated adipocytes were stained with oil red. All reagents were purchased from Sigma, St. Louis, MO, USA. Immunophenotype assays were performed with CD90 and CD45 (all from BD Bioscience Pharmingen, San Diego, CA, USA). The cells were analyzed in a cell cytometer (FACS Calibur, BD). Cells were 93\% positive for CD90 and 2\% negative for CD45. For animal administration, MSC were obtained from passage 4 to 6 .

MSC $\left(2 \times 10^{5}\right.$ cells/animal $)$ were injected into the tail vein of CT and $2 \mathrm{~K}-1 \mathrm{C}$ animals. Injection was done twice, at the $3 \mathrm{rd}$ and 5 th weeks after renal artery clipping. Animals were evaluated at the 6 th week.

\section{Evaluation of Renal Function}

At the end of experimental protocol, 6 weeks after clipping, animals were housed in metabolic cages for 24-hour urine collection for measurements of diuresis and natriuresis. Animals were anesthetized with ketamine and xylazine and blood samples were collected from abdominal aorta. Animals were euthanized by anesthetic overdose and then both stenotic and contralateral kidneys were immediately removed and divided into two parts, one for immunohistochemical analyses as detailed below and the other for molecular biology. Fragments were transferred to a glace plaque on ice for cortex and medulla separation. Fragments were kept on $-80^{\circ} \mathrm{C}$ until use.

Plasma and urinary concentrations of creatinine (Labtest Kit, Lagoa Santa, Brazil) were determined. The urinary osmolarity was measured in an osmometer (Osmometer 3W2, Advanced Instruments, Norwood, MA, USA). The natriuresis was estimated by a selective ion analyzer (Electrolyte Analyzer, Roche, Austria).

Another set of animals was used to determine urinary flow rate and sodium excretion in each kidney. After 6 weeks, animals were anesthetized with sodium thiopental, $60 \mathrm{mg} / \mathrm{kg}$, i.p. (Cristália, Assunção, Brazil) and placed on a temperature-regulated surgical table to maintain 
body temperature at $37^{\circ} \mathrm{C}$. The trachea was cannulated (PE-50 catheter), spontaneous breathing was maintained, and blood pressure was monitored using the PowerLab data acquisition system (AD Instruments, Bella Vista, Australia). Urine samples were collected separately from both ureters cannulated with catheters i.v. $24 \mathrm{GX} 3 / 4^{\prime \prime} 0,7 \times 19 \mathrm{~mm}$. Urine was collected into an oil layer in a graduated 1 -mL pipette and used to calculate the urinary flow rate. Urine samples were used to measure sodium concentration (flame photometer Corning 410C).

\section{Gene Expression Levels}

Total RNA was purified by the phenol and guanidine isothiocyanate-cesium chloride method using an appropriate kit (TRIzol; Gibco BRL, Rockland, MD, USA). Frozen cortex and medulla fragments were homogenized in the Trizol solution and RNA was purified following the manufacturer instructions. Two microgram of total RNA were treated with DNase (RQ1 RNase-free DNase; Promega, Madison, WI, USA) to avoid genomic DNA contamination and reverse transcribed into cDNA by the addition of a mixture containing $0.5 \mathrm{mg} / \mathrm{mL}$ of oligo(dT), $10 \mathrm{~mm}$ of DTT, $0.5 \mathrm{~mm}$ of deoxynucleoside triphosphates (Amersham Pharmacia Biotech, Uppsala, Sweden), and 200 U of reverse transcriptase enzyme (SuperScript RT; Gibco BRL). The obtained cDNA was used to estimate the mRNA expression levels by quantitative realtime PCR (QuantStudio 7TM Flex Real-Time PCR System, Applied Biosystems, USA) using the following specific primers for each molecule (forward and reverse, respectively): $\beta$-actin ( $5^{\prime}$ cct cta tgc caa cac agt gc $3^{\prime}$ and $5^{\prime}$ aca tct gct gga agg tgg ac $3^{\prime}$ ), aquaporin (AQP) 1 ( $5^{\prime}$ tga ccg gcc agg gec tg $3^{\prime}$ and $5^{\prime}$ agc agg tgt cca aga gcc ac $\left.3^{\prime}\right)$, AQP2 ( $5^{\prime}$ cac cgc tcc ttt ttg tct tc $3^{\prime}$ and $5^{\prime}$ gtc ccc acg gat ttc tac tg $3^{\prime}$ ), epithelial sodium channel (ENaC) ( $5^{\prime}$ cca ctg tgg ccg agc tgg tg $3^{\prime}$ and $5^{\prime}$ tca gac tcc atg gtg tcc ag $\left.3^{\prime}\right), \mathrm{Na} / \mathrm{H}$ exchanger (NHE)-3 ( $5^{\prime}$ ttg ggg tcc ttc caa ggc ag $3^{\prime}$ and $5^{\prime}$ cag agt gtc aaa ggg ttc cac $\left.3^{\prime}\right), \mathrm{Na} / \mathrm{K} / 2 \mathrm{Cl}$ cotransporter (NKCC) ( $5^{\prime}$ aga aac ggt gtt cgg gcc tc $3^{\prime}$ and $5^{\prime}$ tct gtc atc cta agt gga cac tg $\left.3^{\prime}\right), \alpha-\mathrm{Na} / \mathrm{K}-\mathrm{ATPase}\left(5^{\prime} \mathrm{ctc}\right.$ tgc ttc gtg ggt ctt atc $3^{\prime}$ and $3^{\prime}$ cac ctg gtt cac tgg aat gt $5^{\prime}$ ). PCR product accumulation was monitored by SYBR Green I intercalating dye (Molecular Probes, Eugene, OR, USA), which exhibits higher fluorescence upon binding of double-stranded DNA. Relative expression of the genes was normalized with the values obtained for the $\beta$-actin expression.

\section{Immunohistochemical Staining}

Half kidneys were fixed with $10 \%$ buffered formaldehyde for $24 \mathrm{~h}$, washed with $70 \%$ ethanol for $24 \mathrm{~h}$, and then embedded in paraffin. Sections were cut with a thickness of $4 \mu \mathrm{m}$. For immunohistochemical procedures, the kidney slices were deparaffinized and rehydrated. To expose the antigens, kidney sections were boiled in a target retrieval solution (citrate buffer $\mathrm{pH}$ 6.0) for $30 \mathrm{~min}$. Endogenous peroxidase activity was blocked with $3 \% \mathrm{H}_{2} \mathrm{O}_{2}$ for 15 min at room temperature. Nonspecific binding was prevented by incubating the sections with a protein blocker (Dako, CA, USA). Sections were incubated overnight at $4{ }^{\circ} \mathrm{C}$ with a primary anti-AQP1 monoclonal antibody (1:100, Sigma, St. Louis, MO, USA) or a primary anti-AQP2 monoclonal antibody (1:50, Abcam, Cambridge, MA, USA). After washing with TBS, the sections previously incubated with primary anti-AQP1 antibody were incubated with horseradish peroxidase-conjugated polymer (Dako, CA, USA) for $30 \mathrm{~min}$ at room temperature. For the primary anti-AQP2 antibody, additional incubation with biotin-conjugated secondary antibody (Zymed Laboratories, Thermo Fisher Scientific, Waltham, MA, USA) was performed for $30 \mathrm{~min}$ at room temperature. Slides were rinsed with PBS, and the sites of antibodyantigen reactions were visualized with 3,3'-diaminobenzidine (Dako, CA, USA). The sections were lightly counterstained with hematoxylin. The analyses were performed using light microscopy (Eclipse 2000 camera Nikon DS-Fi2). Quantification of immunostaining was performed by analyzing five images of the renal cortex and medulla for both kidneys of each 


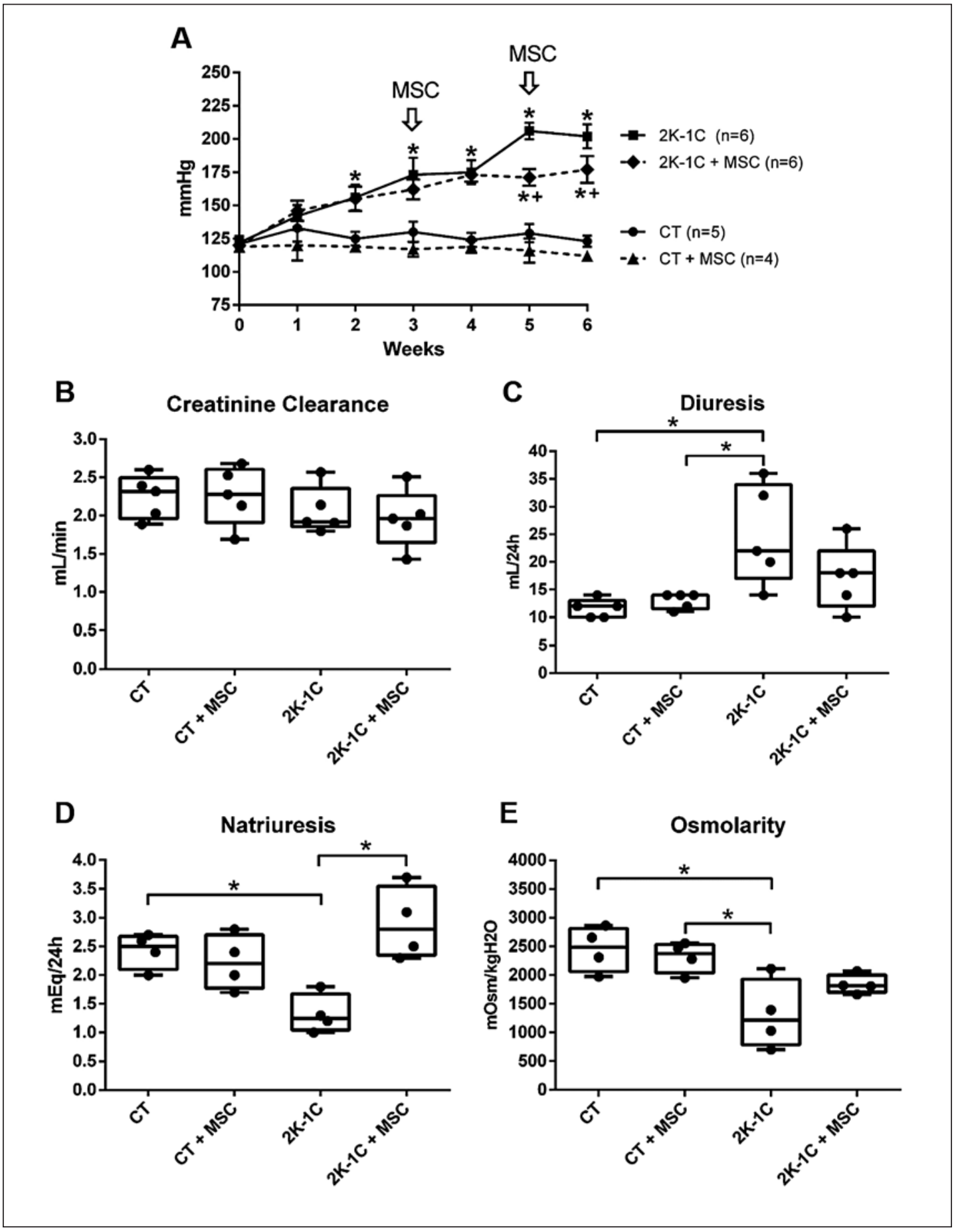

Fig. 1. Effect of MSC on blood pressure and renal function. A Systolic blood pressure was estimated weekly during 6 weeks by plethysmography, and MSC were administered at the 3 rd and 5 th week. $* p<0.05$ versus $\mathrm{CT}$; ${ }^{+}$versus $2 \mathrm{~K}-1 \mathrm{C}+\mathrm{MSC}$. Renal parameters were determined 6 weeks after clamping. Creatinine clearance (B), diuresis (C), natriuresis (D), and urine osmolarity (E) were determined in 24-hour urine samples obtained in the metabolic cages. ${ }^{*} p<0.05$ (B-E). 
A

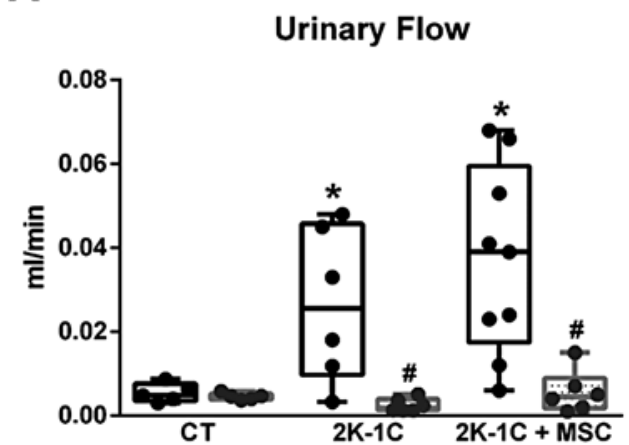

B

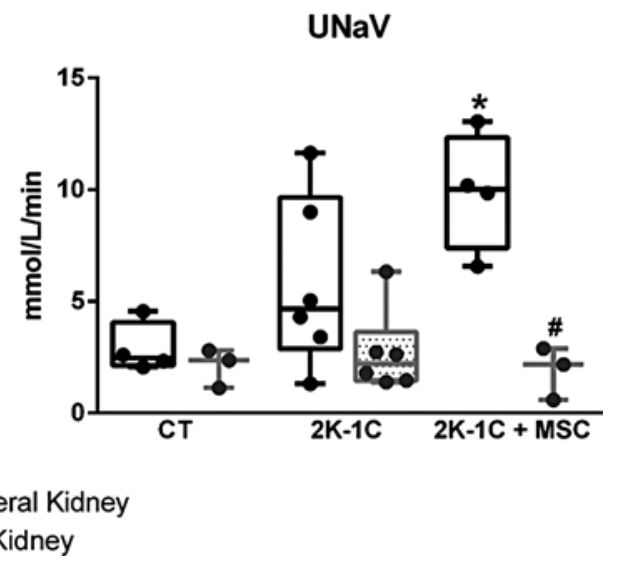

Fig. 2. Urinary flow rate (A) and sodium excretion (UNaV; B) from both kidneys, stenotic and contralateral. ${ }^{*} p<0.05$ versus CT; ${ }^{*} p<0.05$ versus contralateral kidney.

animal and the intensity of staining was quantified using the optical density function of the software (LAS V3.8, Leica microsystems, Wetzlar, Germany) and expressed as percentage of positive pixels in the analyzed area.

\section{Statistical Analysis}

Results are presented as mean \pm standard error. Data were analyzed by two-way ANOVA followed by the Tukey's post-test method using the GraphPad Prism 6 Software (GraphPad Software, San Diego, CA, USA). Statistical significance was defined as $p<0.05$.

\section{Results}

Cell Therapy Prevented the Progressive Increase of Blood Pressure

Baseline levels of SBP were similar among groups (Fig. 1A) and then increased gradually during the 2 weeks after renal artery clipping, and by the 3rd week, SBP was significantly elevated in the $2 \mathrm{~K}-1 \mathrm{C}$ rats compared with the CT group. As observed previously [1, 2], MSC treatment prevented the progressive increase in SBP in contrast to untreated $2 \mathrm{~K}-1 \mathrm{C}$ rats (Fig. 1A).

\section{Cell Therapy Did Not Prevent Polyuria and Potentiate Sodium Excretion}

Creatinine clearance did not differ significantly among groups (Fig. 1B). 2K-1C animals presented increased diuresis compared to the CT group and treatment with MSC produced no significant changes in urine output (Fig. 1C). In contrast $2 \mathrm{~K}-1 \mathrm{C}$ animals presented a reduced capacity to excrete sodium (Fig. 1D) and as a consequence, the urine osmolarity was significantly reduced in the $2 \mathrm{~K}-1 \mathrm{C}$ group (Fig. 1E). Treatment with MSC restored the capacity of hypertensive animals to excrete sodium.

As shown in Figure 2A, the urinary flow rate was similar among stenotic kidneys in all groups. In contrast, the hypertensive contralateral kidney presented a significant increase in diuresis compared with control contralateral kidney. MSC treatment resulted in further rise in the urinary volume. 


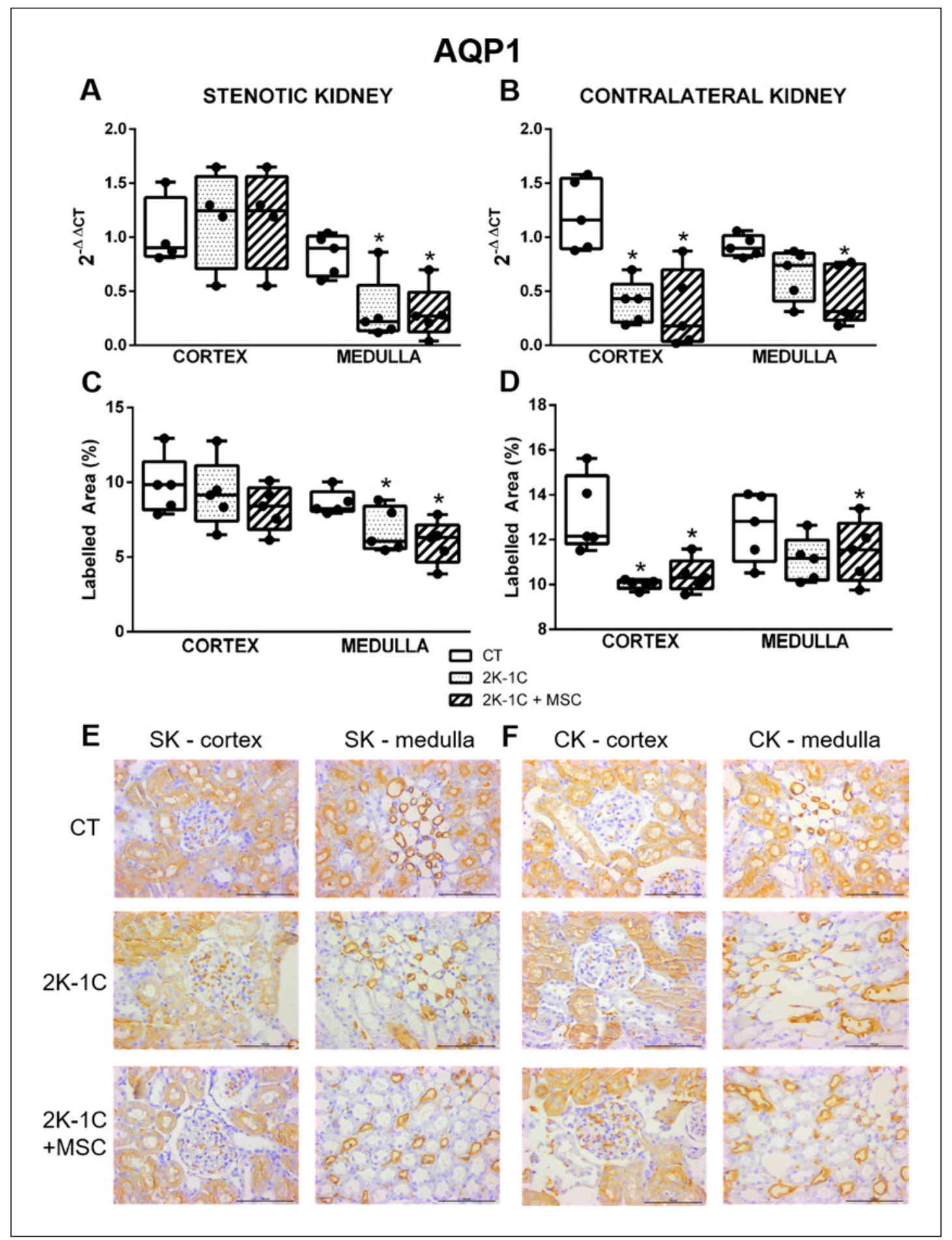

Fig. 3. Expression of aquaporin 1 (APQ1) in both kidneys. A mRNA expression of AQP1 in the cortex and medulla of stenotic kidney. B mRNA expression of AQP1 in the cortex and medulla of contralateral kidney. C Quantification of the labeled area of immunohistochemistry of AQP1 in cortex and medulla of stenotic kidney. D Quantification of the labeled area of immunohistochemistry of AQP1 in cortex and medulla of contralateral kidney. E Representative picture of immunohistochemistry of AQP1 in the cortex and medulla of stenotic kidney (SK). F Representative picture of immunohistochemistry of AQP1 in the cortex and medulla of contralateral kidney (CK). ${ }^{*} p<0.05$ versus CT. 


\section{Kidney \\ Blood Pressure \\ Research}

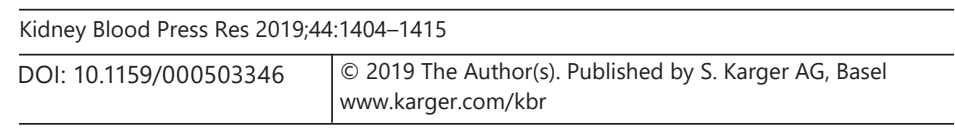

Varela et al.: Mesenchymal Stem Cells and Renovascular Hypertension

The urinary sodium excretion was relatively maintained in stenotic kidney among groups, but sodium excretion was increased in contralateral kidney of the $2 \mathrm{~K}-1 \mathrm{C}$ animals. Sodium loss was potentiated by MSC treatment (Fig. 2B).

\section{Expression of Water Channels in Both Kidneys}

Gene and protein expressions of the water channels were performed separately in cortex and medulla of both stenotic and contralateral kidneys. There was no significant modification in AQP1 gene expression in the cortex of stenotic kidneys, but it was significantly reduced in the medulla (Fig. 3A). AQP1 distribution was evidenced by brown staining in glomeruli, proximal tubule, and descending loop of Henle. AQP1 quantification by immunohistochemistry showed a similar profile of gene expression, i.e., it was decreased only in the medulla of stenotic kidneys (Fig. 3C, E). MSC treatment did not modify this profile in stenotic kidneys. In contrast, AQP1 gene expression was reduced in cortex and medulla of the contralateral kidneys in both $2 \mathrm{~K}-1 \mathrm{C}$ treated and untreated groups (Fig. 3B, D, F). AQP2 mRNA expression in the medulla was similar among groups and between stenotic and contralateral kidneys (Fig. 4A). AQP2 was primarily found in the distal tubules and collecting duct, with no significant changes among groups or between stenotic and contralateral kidneys (Fig. 4B-E).

\section{Renal Sodium Transporters}

The analysis of the gene expression of the major sodium transporters was made respecting the main location of each molecule. No significant changes were observed in Na/K-ATPase, NHE-3, NKCC, and ENaC among groups or between stenotic and contralateral kidneys (Fig. 5).

\section{Discussion}

The hypertensive animals presented an increase in SBP compatible with the model of HRV. MSC treatment prevented the progressive increase in blood pressure after the first administration in the 3rd week post-clamping, reproducing the data recently published by our laboratory [1].

The creatinine clearance calculated using 24-hour urine samples was not changed in $2 \mathrm{~K}-1 \mathrm{C}$ animals, considering that the kidneys are under different perfusion conditions. Maintenance of creatinine clearance may have been a result of a compensatory increase in GFR by the contralateral kidney, since the stenotic kidney was severely damaged by chronic ischemia. However, Oliveira-Sales et al. [2] showed previously that GFR and RPF were reduced in both stenotic and contralateral kidneys and thus, the presence of hyperfiltration in the contralateral kidney was not responsible for the creatinine clearance maintenance, indicating that after 6 weeks, the hypertensive contralateral kidney already shows signs of deterioration of its function. Thus, the maintenance of creatinine clearance observed in awake animals may have been overestimated once we used creatinine as a marker. Hypertensive animals presented increase in diuresis and reduction in natriuresis and, therefore, reduction in urinary osmolarity. These results are consistent with previous data $[1,17]$ and indicate that $2 \mathrm{~K}-1 \mathrm{C}$ animals have a deficit in the urine concentration capacity together with an inability to handling sodium, which in turn could contribute to hypertension. On the other hand, the tubular function (in contrast to the glomerular function) appears to be well preserved in the contralateral kidney. In a previous study, we showed that the polyuria observed in the hypertensive animals occurred through the contralateral kidney, since the stenotic kidney presented a reduced diuresis, suggesting that the contralateral kidney is mainly responsible for the excretion of water in hypertensive animals $[1,2]$. 


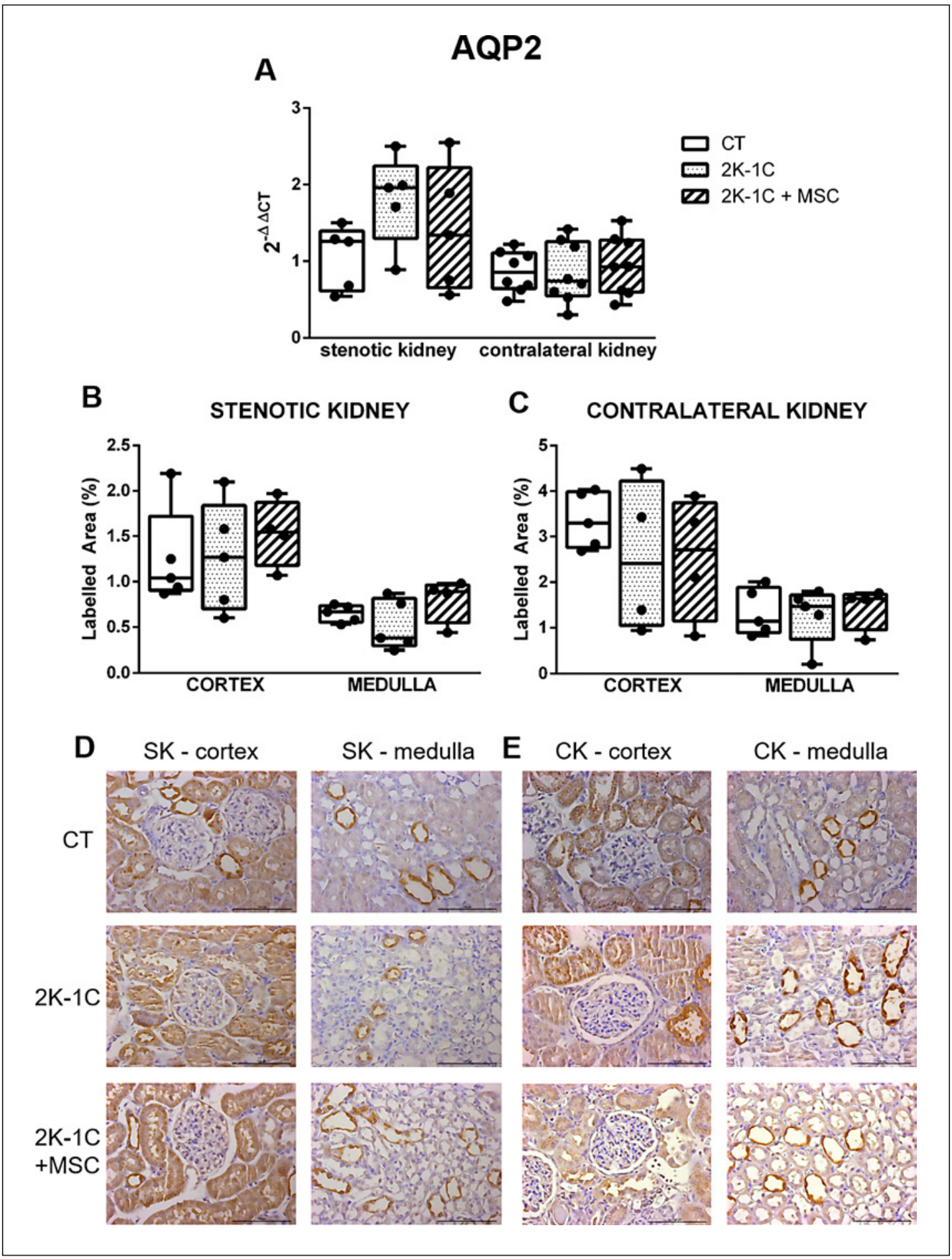

Fig. 4. Expression of aquaporin 2 (AQP2) in both kidneys. A mRNA expression of AQP2 in the medulla of stenotic kidney and contralateral kidney. B Quantification of the labeled area of immunohistochemistry of AQP2 in cortex and medulla of stenotic kidney. C Quantification of the labeled area of immunohistochemistry of AQP2 in cortex and medulla of contralateral kidney. D Representative picture of immunohistochemistry of AQP2 in the cortex and medulla of stenotic kidney (SK). E Representative picture of immunohistochemistry of AQP2 in the cortex and medulla of contralateral kidney (CK). 


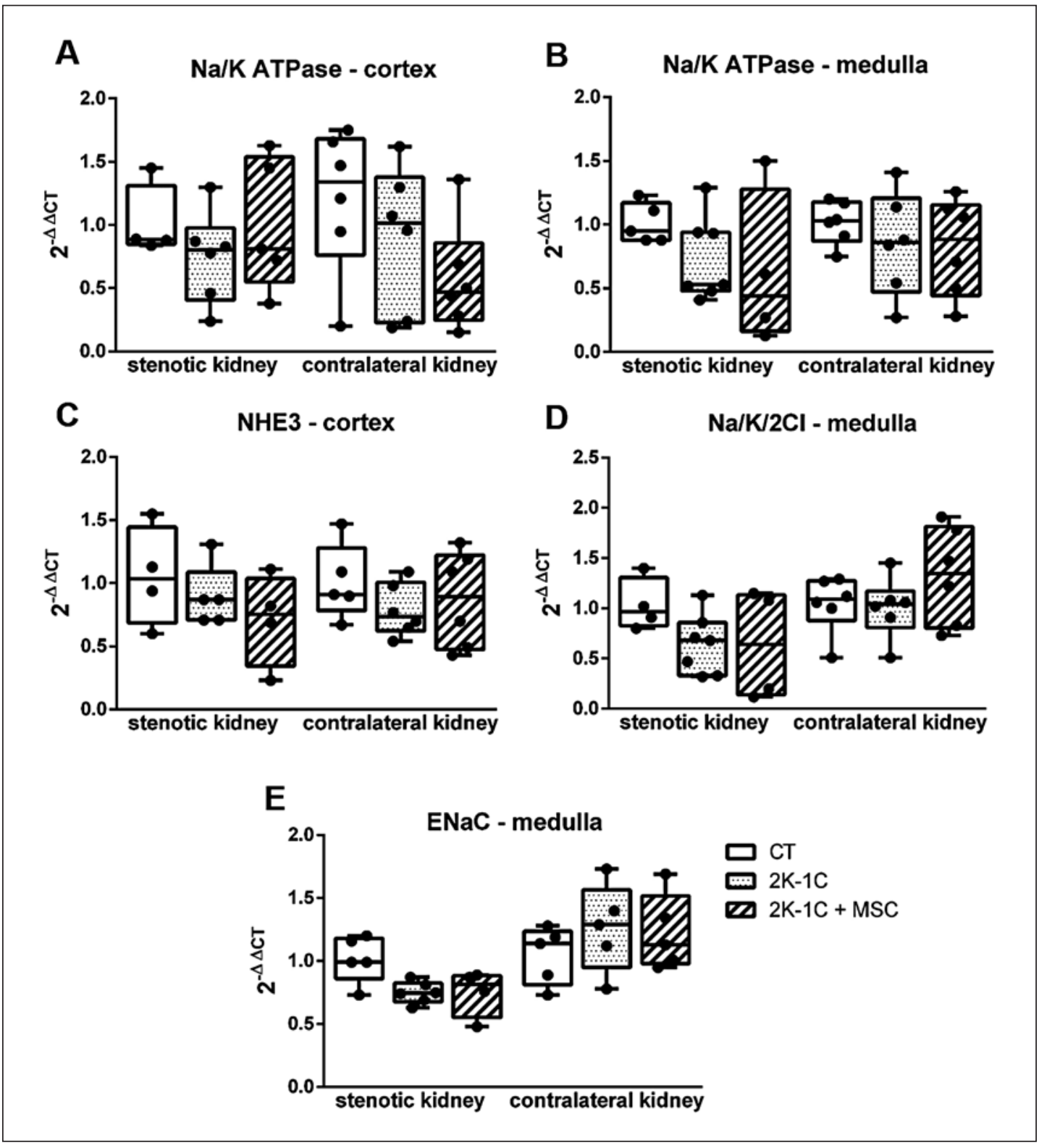

Fig. 5. Gene expression of the main renal sodium transporters in both kidneys, stenotic kidney and contralateral kidney. A Na/K-ATPase in cortex. B Na/K-ATPase in medulla. C NHE-3 in cortex. D Na/K/2Cl in medulla. E ENaC in medulla.

In the present study, there was an increase in water excretion by the contralateral kidney. Considering that the levels of AQP1 but not of AQP2 were reduced in the contralateral kidney, one can suggest that AQP1 suppression may be, at least in part, responsible for polyuria of the contralateral kidney and therefore, the increase in diuresis occurred independently of the antidiuretic hormone. In addition, it was previously observed that plasma levels of antidiuretic hormone were unchanged in 2K-1C animals [18]. Studies by Ma et al. [19] also showed suppression of AQP1 in stenotic kidney, but, in contrast to our results, they found no change in the contralateral kidney. This discrepancy probably reflects different phases of the experimental design, since Ma et al.'s study used a 1-week protocol and here, we used 6 weeks. Moreover, it is important to emphasize that after 1 week, the contralateral kidney probably 


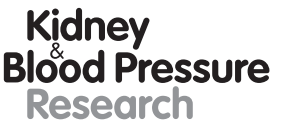

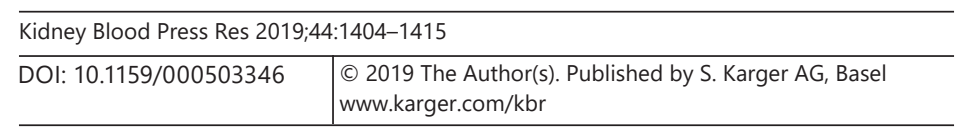

Varela et al.: Mesenchymal Stem Cells and Renovascular Hypertension

did not completely adapt to hypertension. Taken together, these results suggest that AQP1 is the main water transporter related to the reduction of urinary osmolarity and increased diuresis in this RVH model. The mechanism by which AQP1 was decreased was not analyzed in this study. However, microvascular rarefaction because of chronic hypoxia may compromise the integrity of the vessels and decrease the oxygenation of the medulla, which could interfere with the expression of AQP1 affecting urinary concentration $[1,13,14]$.

The total sodium excretion was diminished in hypertensive animals; however, when analyzed separately, the sodium excretion by the stenotic kidney was reduced, whereas it was elevated by the contralateral kidney. Considering that the contralateral kidney is a hypertensive kidney, its increased capacity to excrete sodium has been attributed to the pressure natriuresis [20]. In fact, Mackenzie et al. [8] demonstrated that the reduction of perfusion pressure to the nonclipped kidney of $2 \mathrm{~K}-1 \mathrm{C}$ animals to normal levels resulted in reduced sodium excretion. In the present study, we did not find significant changes in the expression of the main sodium transporters either in untreated or MSC-treated animals. However, the role of these transporters in the increased natriuresis exhibited by the contralateral kidney cannot be excluded, since the protein levels were not analyzed in the present study. In fact, Lira et al. [21] demonstrated that a single intracapsular injection of MSCs improved the activity of $\mathrm{Na}$ /K-ATPase mainly in the clipped kidney, increasing the tubular reabsorption capacity. These discrepancies could be related to the differences in the route of MSCs administration, since Lira and colleagues used one kidney subcapsular MSC injection at day 4 after clipping and we used twice intravenous injection, which allows MSC to reach both kidneys in contrast to subcapsular injection, which restricts MSC to the stenotic kidney. On the other hand, it is important to consider that the reduction in the AQP1 mRNA levels in both cortex and medulla of the contralateral kidney would impair the countercurrent mechanism and reduce sodium reabsorption by ascending limb. Thus, together with pressure natriuresis, the impairment of the countercurrent mechanism could contribute to high natriuresis observed in the present study.

\section{Conclusion}

After 6 weeks of left renal artery clamping, the contralateral hypertensive kidney showed signs of functional deterioration, but with preserved ability to excrete sodium and water, mainly at the expense of pressure natriuresis and reduction of AQP1 expression. MSC acts by a mechanism not yet fully known, but it was effective in reducing SBP without altering the ability of the hypertensive kidney to excrete sodium and water, thus preventing the expansion of extracellular volume that contributes to worse hypertension.

\section{Statement of Ethics}

All experimental procedures were approved by the Ethics in Research Committee of the Federal University of Sao Paulo (434506/13).

\section{Disclosure Statement}

The authors have no conflicts of interest to declare. 


\section{Kidney \\ Blood Pressure \\ Research}

\begin{tabular}{l|l}
\hline Kidney Blood Press Res 2019;44:1404-1415 \\
\hline DOI: 10.1159/000503346 & $\begin{array}{l}\text { @ 2019 The Author(s). Published by S. Karger AG, Basel } \\
\text { www.karger.com/kbr }\end{array}$ \\
\hline
\end{tabular}

Varela et al.: Mesenchymal Stem Cells and Renovascular Hypertension

\section{Funding Sources}

This work was supported by grants from the Coordenação de Aperfeiçoamento de Nível Superior (CAPES), Fundação de Amparo à Pesquisa do Estado de São Paulo (FAPESP 2010/52180-4), and Conselho Nacional de Desenvolvimento Científico e Tecnológico (CNPq - 470662/2013-1).

\section{Author Contributions}

V.A.V.: data acquisition, conducting the experiments, analysis, interpretation, discussion, and manuscript writing. E.B.O.-S., E.M., F.T.B., P.P.G., A.d.S.N., and C.G.S.: conducting the experiments, data interpretation, and discussion. M.A.B.: study conception and design, data analysis and interpretation, financial support, discussion, reviewing, and editing the manuscript.

\section{References}

1 Oliveira-Sales EB, Maquigussa E, Semedo P, Pereira LG, Ferreira VM, Câmara NO, et al. Mesenchymal stem cells (MSC) prevented the progression of renovascular hypertension, improved renal function and architecture. PLoS One. 2013 Nov;8(11):e78464.

2 Oliveira-Sales EB, Varela VA, Maquigussa E, Borges FT, Shimoura CG, Gomes G, et al. Renovascular hypertension: effects of mesenchymal stem cells in the contralateral hypertensive kidney in rats. Clin Exp Hypertens. 2016; 38(7):586-93.

3 Campos RR, Oliveira-Sales EB, Nishi EE, Paton JF, Bergamaschi CT. Mechanisms of renal sympathetic activation in renovascular hypertension. Exp Physiol. 2015 Apr;100(5):496-501.

4 Martinez-Maldonado M. Pathophysiology of renovascular hypertension. Hypertension. 1991 May;17(5):707-19.

5 Safian RD, Textor SC. Renal-artery stenosis. N Engl J Med. 2001 Feb;344(6):431-42.

6 Navar LG, Zou L, Von Thun A, Tarng Wang C, Imig JD, Mitchell KD. Unraveling the Mystery of Goldblatt Hypertension. News Physiol Sci. 1998 Aug;13:170-6.

7 Tafur-Soto JD, White CJ. Renal artery stenosis. Cardiol Clin. 2015 Feb;33(1):59-73.

8 Mackenzie HS, Morrill AL, Ploth DW. Pressure dependence of exaggerated natriuresis in two-kidney, one clip Goldblatt hypertensive rats. Kidney Int. 1985 May;27(5):731-8.

9 Rostand SG, Kirk KA. Attenuated pressure natriuresis in the early phases of two-kidney Goldblatt hypertension. Am J Physiol. 1984 May;246(5 Pt 2):F691-9.

10 Mai M, Geiger H, Hilgers KF, Veelken R, Mann JF, Dämmrich J, et al. Early interstitial changes in hypertensioninduced renal injury. Hypertension. 1993 Nov;22(5):754-65.

11 Ploth DW, Roy RN, Huang WC, Navar LG. Impaired renal blood flow and cortical pressure autoregulation in contralateral kidneys of Goldblatt hypertensive rats. Hypertension. 1981 Jan-Feb;3(1):67-74.

12 Steiner RW, Tucker BJ, Gushwa LC, Gifford J, Wilson CB, Blantz RC. Glomerular hemodynamics in moderate Goldblatt hypertension in the rat. Hypertension. 1982 Jan-Feb;4(1):51-7.

13 Chade AR, Kelsen S. Renal microvascular disease determines the responses to revascularization in experimental renovascular disease. Circ Cardiovasc Interv. 2010 Aug;3(4):376-83.

14 Chade AR. Renal vascular structure and rarefaction. Compr Physiol. 2013 Apr;3(2):817-31.

15 Ruilope L, Garcia-Robles R, Sancho-Rof J, Paya C, Rodicio JL, Strong CG, et al. Effect of furosemide on renal function in the stenotic and contralateral kidneys of patients with renovascular hypertension. Hypertension. 1983 Nov-Dec; $5(6$ Pt 3):V43-7.

16 Goldblatt H, Lynch J, Hanzal RF, Summerville WW. STUDIES ON EXPERIMENTAL HYPERTENSION : I. THE PRODUCTION OF PERSISTENT ELEVATION OF SYSTOLIC BLOOD PRESSURE BY MEANS OF RENAL ISCHEMIA. J Exp Med. 1934 Feb;59(3):347-79.

17 Oliveira-Sales EB, Varela VA, Maquigussa E, Borges FT, Shimoura CG, Gomes G, et al. Renovascular hypertension: effects of mesenchymal stem cells in the contralateral hypertensive kidney in rats. Clin Exp Hypertens. 2016; 38(7):586-93.

18 Rabito SF, Carretero OA, Scicli AG. Evidence against a role of vasopressin in the maintenance of high blood pressure in mineralocorticoid and renovascular hypertension. Hypertension. 1981 Jan-Feb;3(1):34-8.

19 Ma SK, Bae EH, Kim IJ, Choi C, Lee J, Kim SW. Altered renal expression of aquaporin water channels and sodium transporters in rats with two-kidney, one-clip hypertension. Kidney Blood Press Res. 2009;32(6):411-20.

20 Pickering TG. Renovascular hypertension: etiology and pathophysiology. Semin Nucl Med. 1989 Apr;19(2): 79-88.

21 Lira R, Oliveira M, Martins M, Silva C, Carvalho S, Stumbo AC, et al. Transplantation of bone marrow-derived MSCs improves renal function and $\mathrm{Na}++\mathrm{K}+-\mathrm{ATPase}$ activity in rats with renovascular hypertension. Cell Tissue Res. 2017 Aug;369(2):287-301. 